\title{
Brugada syndrome in childhood: a potential fatal arrhythmia not always recognised by paediatricians. A case report and review of the literature
}

Received: 22 January 2006 / Revised: 17 March 2006 / Accepted: 23 March 2006 / Published online: 3 May 2006

(C) Springer-Verlag 2006

\begin{abstract}
We report on a youngster followed by his paediatrician from birth until 14 years of age for premature beats, most likely of ventricular origin. The sudden death of his sister provoked a re-assessment of his electrocardiograms (ECG), resulting in the diagnosis of Brugada syndrome and the subsequent implantation of a cardioverter defibrillator. This syndrome is a well known entity in adult cardiology, first described by Brugada and Brugada in 1992. It is considered to be the second most common cause of death in young adults after road traffic accidents. In children, however, the Brugada syndrome is not well known and we believe to be certainly underdiagnosed.
\end{abstract}

Keywords Brugada syndrome $\cdot$ Child - Syncope Sudden death $\cdot$ Bundle branch block $\cdot$ Ventricular fibrillation

Abbreviations ECG: Electrocardiogram - EP study: Electrophysiological study $\cdot$ ICD: Implantable cardioverter defibrillator - RBBB: Right bundle branch block

\section{Introduction}

The Brugada syndrome, as described by Brugada and Brugada in 1992, is a cardiac electrical disease which includes an atypical right bundle branch block (RBBB) pattern with ST-T segment alterations [9]. Over the last 10 years, this syndrome has received a great deal of attention in cardiology literature, due to its association with

\footnotetext{
Y. Mivelaz $(\bowtie) \cdot$ S. Di Bernardo ·

E. J. Meijboom · N. Sekarski

Paediatric Cardiology, Lausanne University Hospital, CHUV, Rue du Bugnon 46,

CH-1011 Lausanne, Switzerland

e-mail: Yvan.Mivelaz@chuv.ch

Tel.: +41-21-3143691

Fax: +41-21-3143665

\section{E. Pruvot}

Cardiology, Lausanne University Hospital,

CHUV, Rue du Bugnon 46,

CH-1011 Lausanne, Switzerland
}

sudden death. Reports of Brugada syndrome in the paediatric literature, however, remain limited to a small number of publications [2, 20, 23, 24, 27, 30].

Although it is generally assumed to be uncommon in the paediatric population, one should exclude the Brugada syndrome in a child with a family history of unexplained sudden death [30].

\section{Case Report}

Our patient was born following a normal pregnancy and delivery in 1991. His physical examination was normal, except for an irregular cardiac rhythm. He was referred to a paediatric cardiologist because his electrocardiogram (ECG) showed right-axis deviation $\left(190^{\circ}\right)$ and repetitive monomorphic premature beats of likely ventricular origin (Fig. 1). His echocardiogram was normal and he was followed on a 3 -month basis.

His ECG at 3 months remained abnormal, but differed from the one at birth (Fig. 2, panel A). The ECG showed an RBBB pattern with deep negative T-waves in the right precordial leads. The child remained asymptomatic and was followed regularly. A progressive reduction in the amplitude of the RBBB was noted (Fig. 2, panel B and C).

In 2004, his previously healthy 20 -year-old sister died suddenly after a meal without any explanation on autopsy. The brother was then referred to our Paediatric Cardiology Unit for re-evaluation.

He had remained asymptomatic, but his baseline ECG (Fig. 3) now showed a "coved-type" RBBB pattern with deep negative T-waves in the right precordial leads [3].

Echocardiogram and cardiac-MRI did not show any structural anomalies. Holter recording and stress testing did not report any arrhythmia. Nevertheless, the intravenous infusion of Ajmaline (a class IA sodium channel blocker) at increasing dose enhanced the repolarisation anomalies in leads V1 and V2, as reported in Brugada syndrome. An electrophysiology (EP) study was then performed, which showed inducible polymorphic ventricular tachycardia. Because of his positive family history, spontaneous type-1 
Fig. 1 ECG V1 and V2 precordial leads showing monomorphic premature beats (duration $120 \mathrm{~ms}$ ) in trigeminy (arrows). The absence of $\mathrm{P}$-waves preceding aberrant complexes suggests a ventricular origin. However, a supraventricular origin cannot be excluded
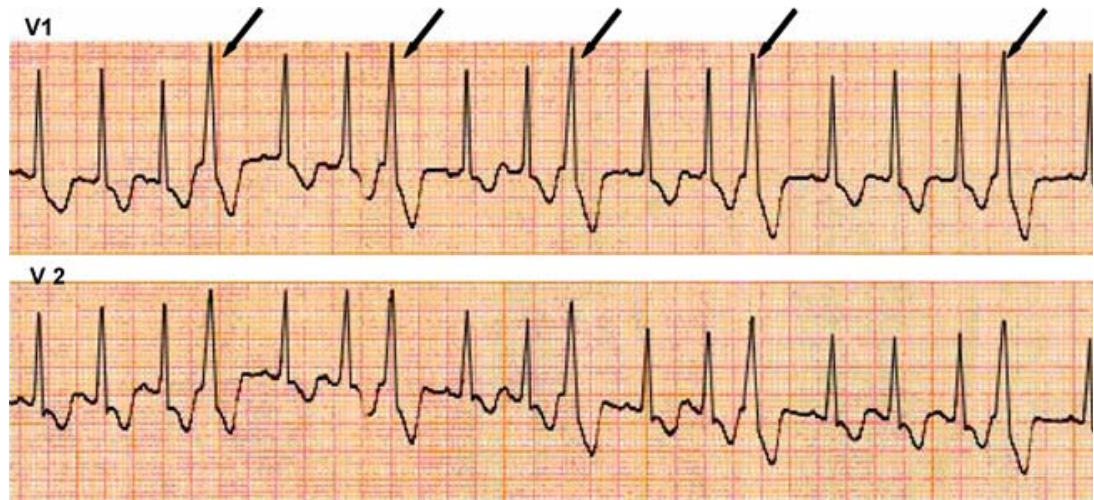

Brugada pattern and inducibility at EP study, the patient was considered at high risk for sudden death and underwent implantation of an implantable cardioverter defibrillator (ICD).

The first- and second-degree relatives of the family were then screened (clinical evaluation and an ECG). Two additional members (his mother and a maternal uncle) with suggestive ECG pattern of Brugada syndrome were further investigated (Ajmaline test) and were diagnosed with Brugada syndrome and also implanted with an ICD. The family underwent genetic testing, but the results are still pending.

\section{Discussion}

This case report illustrates a typical case of Brugada syndrome in children: an asymptomatic child with a structurally normal heart showing pre-existent ECG anomalies (premature beats and atypical RBBB) and sudden unexpected death of a close relative.

The Brugada syndrome is mainly a disease of the young adult, with a mean age at sudden death of $41 \pm 15$ years, but also includes patients as young as a few days old $[22,23$, 25]. It is the second leading cause of death after road traffic accidents in young adults [3]. However, its prevalence is
Fig. 2 ECG V1 and V2 precordial leads at 3 months, 4 and 9 years old (A, B and C, respectively) showing $\mathrm{RBBB}$ pattern and ST segment anomalies. Panel A: ECG at 3 months remained abnormal, but differed from the one at birth. The ECG showed an RBBB pattern with deep negative T-waves in the right precordial leads. Panel B and $\mathrm{C}$ : A progressive reduction in the amplitude of the RBBB is noted. However, panel C clearly shows some slurring of the downslope of the $\mathrm{R}^{\prime}$-wave (arrows) and persistence of an overt negative T-wave

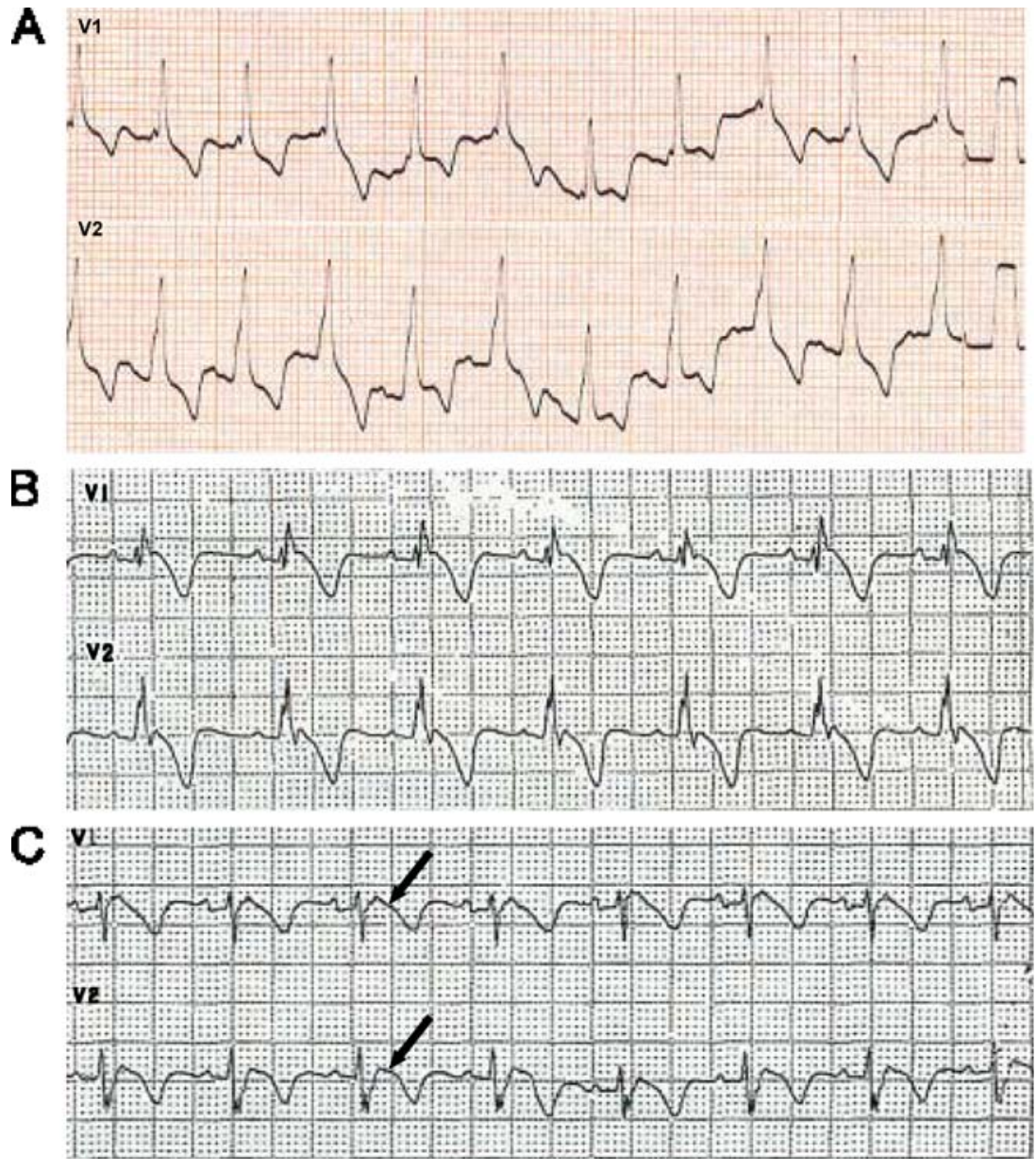


Fig. 3 ECG V1 and V2 precordial leads at 13 years old showing typical aspect of a "coved-type," or type-1 ECG pattern of Brugada syndrome: a "coved-type" ST segment elevation with a negative T-wave in the right precordial leads. Note the overt slurring (arrows) in the downslope of the $\mathrm{R}^{\prime}$-wave

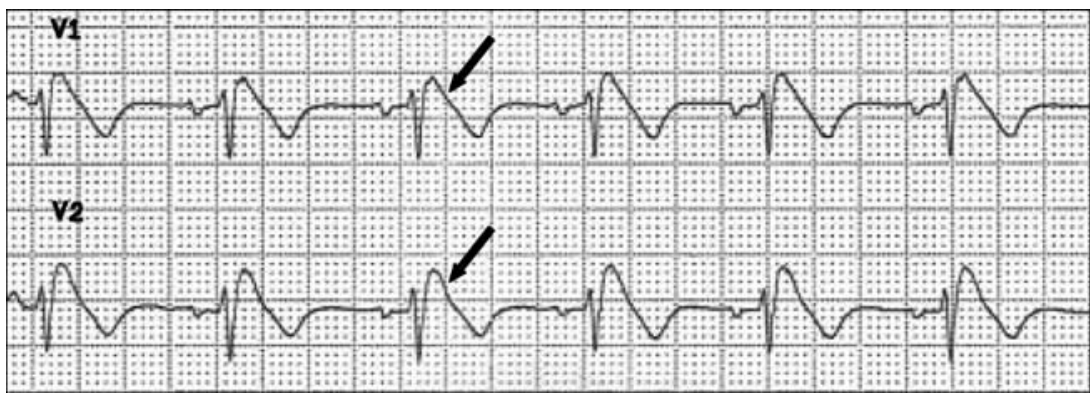

difficult to assess because of the dynamic aspect of the ECG pattern, influenced by many parameters, but also because of the difference in the criteria which are used to define it. Prevalence ranges from 5 per 10,000 inhabitants up to 70 per 10,000 inhabitants, with the highest prevalence in south-east Asia [3, 16].

It is primarily an electrical disease involving cardiac sodium channels, as in long QT syndrome type-3. Some overlap between the two diseases exists. Both are associated with mutations in the $S C N 5 A$ gene, but at different sites of the gene [7]. The SCN5A gene is coding for the $\alpha$-subunit of the cardiac sodium channel; it is the only gene found to be linked to Brugada syndrome. Inheritance occurs via an autosomal dominant mode of transmission and more than 80 mutations have been discovered, leading to many different phenotypes. Curiously, a male preponderance of the ECG phenotype (8 to 10 times more prevalent) is observed, but is not yet completely understood, although sex hormones seem to play a significant role $[11,14,31]$. SCN5A mutations, however, are found in only $18 \%$ to $30 \%$ of Brugada syndromes and, thus, are of limited help for the screening, diagnosis and management of the disease [3].

These abnormal channels have an altered function which reduces the inward sodium current, inducing conduction delay and enhancing susceptibility to re-entrant ventricular arrhythmias [13]. Nevertheless, the strong assumption that the Brugada syndrome is an electric disorder, without any functional and structural cardiac abnormalities, has been recently challenged by Frustaci et al. [12]. They detected structural alterations on endomyocardial biopsy (localised right ventricular myocarditis, some with detectable viral genomes, right ventricular cardiomyopathy or cardiomyopathic changes) in all of their 18 patients with Brugada syndrome. On this basis, they suggest that the Brugada syndrome is not purely an electrical disease but, rather, a common electrical manifestation of structural abnormalities in the right ventricle resulting from different origins: genetic, infectious and inflammatory. However, these findings will need confirmation.

The conduction disorder resulting from sodium channel alteration is responsible for the ECG patterns associated with Brugada syndrome. Only one of these patterns is diagnostic of Brugada syndrome, known as type 1 ECG pattern or the "coved-type" (Fig. 3). Other types of ECG pattern, type 2 and type 3 , tend to be grouped as the "saddle-back type", an intermediate pattern that requires conversion into the "coved-type" after pharmacological challenge or confirmation by genetic analysis to be diagnostic of Brugada syndrome [15].

Sodium channel blockers are used to unmask transient Brugada ECG patterns or demonstrate conversion of a "saddle-back" to a "coved-type" ECG pattern. Four of them are currently used: Ajmaline, Fleicanide, Procainamide and Pilsicainide [3,29]. Cautious administration should include haemodynamic monitoring, continuous 12-leads ECG recording and the availability of appropriate resuscitation equipment.

Apart from sodium-channel blockers, many other conditions are reported to help unmask Brugada syndrome phenotype. Some drugs (anti-arrhythmics, anti-anginal, psychotrops), electrolyte imbalances (hyper- and hypokalaemia, hypercalcaemia) and febrile state are the most common $[4,18]$.

A "coved-type" ECG pattern in more than one right precordial lead $(\mathrm{V} 1-\mathrm{V} 3)$, in the presence or absence of sodium-channel blockers, without any clinical symptom is not labelled Brugada syndrome but, instead, idiopathic Brugada ECG pattern. The syndrome should be considered only in association with the following criteria: documented ventricular fibrillation, polymorphic ventricular tachycardia, family history of sudden cardiac death at less than 45 years of age, inducibility of ventricular fibrillation with EP study, "coved-type" ECG pattern in family members, syncopal episodes or nocturnal agonal respiration [29].

The spectrum of clinical presentation and prognosis in Brugada syndrome varies widely from asymptomatic patients with normal ECG to symptomatic ones (syncope, seizure, nocturnal agonal respiration) with "coved-type" ECG pattern and sudden death occurring more commonly during sleep. The most challenging task when facing Brugada syndrome concerns the risk stratification of sudden death in asymptomatic patients. Unfortunately, many subjects remain asymptomatic until their fatal event. With the second consensus conference on the Brugada syndrome, a risk stratification scheme has been proposed based on a set of parameters, including presence of symptoms (aborted sudden death, syncope, seizure, nocturnal agonal respiration), spontaneous or drug-induced "coved-type" ECG pattern and positive familial history of sudden death [3]. The utility of EP study as a screening tool has been recently challenged [19]. For those resuscitated from sudden death or at high risk, implantation of an ICD is currently the only proven effective therapy [8, 10]. Prevention of ventricular fibrillation occurring spontaneously or during EP study has been reported in certain 
cases using different drugs, such as quinidine sulphate, cilostazol and isoproterenol, but these findings deserve further validation before being part of the standard management strategy for Brugada syndrome $[1,6,21$, $26,28]$.

In the literature, Brugada syndrome in children was first described in 2000 [20]. Since then, many other cases have been described. To date, there are only three Japanese studies reporting the prevalence and the clinical course of Brugada syndrome in the asymptomatic paediatric population $[17,30,31]$. Interestingly, the prevalence of evocative ECG pattern of Brugada syndrome is much lower than in adults. It increases with age, from 3 per 10,000 children at a mean age of 12 years to 10 per 10,000 children at a mean age of 15 years. The highly dynamic nature of the ECG pattern is well known, but is even more pronounced in children $[5,11,14,31]$. However, the Brugada syndrome could lead to death or near-miss sudden death, even when ECG pattern is only transient. Recently, its possible link with sudden infant death syndrome has been shown with the case of a 19-day-old infant resuscitated from ventricular fibrillation, without QT prolongation nor ST segment anomaly on ECG, but with one of the mutations in the SCN5A gene already described in the Brugada syndrome [23].

The most difficult task lies in establishing the optimal management of suspected and established Brugada syndrome in the paediatric population. To date, only Sreeram et al. have tackled this problem [24]. When a teenager presents a "coved-type" ECG pattern and missed sudden death, attitude should not differ from adults with ICD implantations. In infants and young children, further discussion is required. ICD implantation is not benign at this age and will be required for the rest of the patient's life. The diagnosis and therapeutic strategy is still more complex when there is only a suspicion of Brugada syndrome, for example, when an asymptomatic girl presents a spontaneous but transient type-1 ECG pattern without family history of sudden cardiac death or when a boy with a family history of Brugada or near-miss sudden death does not have a type-1 ECG pattern. For these cases, the question remains if we should follow adult recommendations or deviate. The highly dynamic nature of Brugada syndrome ECG patterns, the risk/benefit ratio of ICD and the weaker probability of sudden death in children must be taken into account and requires an adaptation of these recommendations.

\section{Conclusion}

The Brugada syndrome is a genetic cardiac disease whose first manifestation is often sudden death, affecting otherwise asymptomatic young adults. Even though common manifestation of the Brugada syndrome is in the $40 \mathrm{~s}$, paediatric patients might also be affected with some uncertainty about their prognosis. Thus, paediatricians should be aware of the existence of Brugada syndrome, especially in the context of sudden death amongst close relatives and in cases of unexplained syncope or aborted sudden death. To offer adequate management and followup for a child with suspected Brugada syndrome, risk stratification and therapeutic recommendations based on the specificity of the Brugada syndrome in children must be established.

\section{References}

1. Alings M, Dekker L, Sadee A, Wilde A (2001) Quinidine induced electrocardiographic normalization in two patients with Brugada syndrome. Pacing Clin Electrophysiol 24(9 Pt 1): $1420-1422$

2. Antzelevitch C (2001) Molecular biology and cellular mechanisms of Brugada and long QT syndromes in infants and young children. J Electrocardiol 34(Suppl):177-181

3. Antzelevitch C, Brugada P, Borggrefe M, Brugada J, Brugada R, Corrado D, Gussak I, LeMarec H, Nademanee K, Perez Riera AR, Shimizu W, Schulze-Bahr E, Tan H, Wilde A (2005) Brugada syndrome: report of the second consensus conference: endorsed by the Heart Rhythm Society and the European Heart Rhythm Association. Circulation 111(5):659-670

4. Antzelevitch C, Brugada R (2002) Fever and Brugada syndrome. Pacing Clin Electrophysiol 25(11):1537-1539

5. Beaufort-Krol GC, van den Berg MP, Wilde AA, van Tintelen JP, Viersma JW, Bezzina CR, Bink-Boelkens MT (2005) Developmental aspects of long QT syndrome type 3 and Brugada syndrome on the basis of a single SCN5A mutation in childhood. J Am Coll Cardiol 46(2):331-337

6. Belhassen B, Glick A, Viskin S (2004) Efficacy of quinidine in high-risk patients with Brugada syndrome. Circulation 110 (13): 1731-1737

7. Bezzina C, Veldkamp MW, van Den Berg MP, Postma AV, Rook MB, Viersma JW, van Langen IM, Tan-Sindhunata G, Bink-Boelkens MT, van Der Hout AH, Mannens MM, Wilde AA (1999) A single $\mathrm{Na}(+)$ channel mutation causing both longQT and Brugada syndromes. Circ Res 85(12):1206-1213

8. Brugada J, Brugada R, Brugada P (2000) Pharmacological and device approach to therapy of inherited cardiac diseases associated with cardiac arrhythmias and sudden death. J Electrocardiol 33 (Suppl):41-47

9. Brugada P, Brugada J (1992) Right bundle branch block, persistent ST segment elevation and sudden cardiac death: a distinct clinical and electrocardiographic syndrome. A multicenter report. J Am Coll Cardiol 20(6):1391-1396

10. Brugada P, Brugada R, Brugada J, Geelen P (1999) Use of the prophylactic implantable cardioverter defibrillator for patients with normal hearts. Am J Cardiol 83(5B):98D-100D

11. Di Diego JM, Cordeiro JM, Goodrow RJ, Fish JM, Zygmunt AC, Perez GJ, Scornik FS, Antzelevitch C (2002) Ionic and cellular basis for the predominance of the Brugada syndrome phenotype in males. Circulation 106(15):2004-2011

12. Frustaci A, Priori SG, Pieroni M, Chimenti C, Napolitano C, Rivolta I, Sanna T, Bellocci F, Russo MA (2005) Cardiac histological substrate in patients with clinical phenotype of Brugada syndrome. Circulation 112(24):3680-3687

13. Juang JM, Huang SK (2004) Brugada syndrome-an underrecognized electrical disease in patients with sudden cardiac death. Cardiology 101(14):157-169

14. Matsuo K, Akahoshi M, Seto S, Yano K (2003) Disappearance of the Brugada-type electrocardiogram after surgical castration: a role for testosterone and an explanation for the male preponderance. Pacing Clin Electrophysiol $26(7 \mathrm{Pt} 1): 1551-1553$

15. Meregalli PG, Wilde AA, Tan HL (2005) Pathophysiological mechanisms of Brugada syndrome: depolarization disorder, repolarization disorder, or more? Cardiovasc Res 67(3):367-378 
16. Miyasaka Y, Tsuji H, Yamada K, Tokunaga S, Saito D, Imuro Y, Matsumoto N, Iwasaka T (2001) Prevalence and mortality of the Brugada-type electrocardiogram in one city in Japan. J Am Coll Cardiol 38(3):771-774

17. Oe H, Takagi M, Tanaka A, Namba M, Nishibori Y, Nishida Y, Kawarabayashi T, Yoshiyama M, Nishimoto M, Tanaka K, Yoshikawa J (2005) Prevalence and clinical course of the juveniles with Brugada-type ECG in Japanese population. Pacing Clin Electrophysiol 28(6):549-554

18. Porres JM, Brugada J, Urbistondo V, Garcia F, Reviejo K, Marco P (2002) Fever unmasking the Brugada syndrome. Pacing Clin Electrophysiol 25(11):1646-1648

19. Priori SG, Napolitano C (2005) Should patients with an asymptomatic Brugada electrocardiogram undergo pharmacological and electrophysiological testing? Circulation 112 (2):279-292; discussion 279-292

20. Priori SG, Napolitano C, Giordano U, Collisani G, Memmi M (2000) Brugada syndrome and sudden cardiac death in children. Lancet 355(9206):808-809

21. Probst V, Evain S, Gournay V, Marie A, Schott JJ, Boisseau P, LeMarec H (2006) Monomorphic ventricular tachycardia due to brugada syndrome successfully treated by hydroquinidine therapy in a 3-year-old child. J Cardiovasc Electrophysiol 17 (1):97-100

22. Sanatani S, Mahkseed N, Vallance H, Brugada R (2005) The Brugada ECG pattern in a neonate. J Cardiovasc Electrophysiol 16(3):342-344

23. Skinner JR, Chung SK, Montgomery D, McCulley $\mathrm{CH}$, Crawford J, French J, Rees MI (2005) Near-miss SIDS due to Brugada syndrome. Arch Dis Child 90(5):528-529

24. Sreeram N, Simmers T, Brockmeier K (2004) The Brugada syndrome. Its relevance to paediatric practice. Z Kardiol 93 (10):784-790
25. Suzuki H, Torigoe K, Numata O, Yazaki S (2000) Infant case with a malignant form of Brugada syndrome. J Cardiovasc Electrophysiol 11(11):1277-1280

26. Tanaka H, Kinoshita O, Uchikawa S, Kasai H, Nakamura M, Izawa A, Yokoseki O, Kitabayashi H, Takahashi W, Yazaki Y, Watanabe N, Imamura H, Kubo K (2001) Successful prevention of recurrent ventricular fibrillation by intravenous isoproterenol in a patient with Brugada syndrome. Pacing Clin Electrophysiol 24(8 Pt 1):1293-1294

27. Towbin JA (2004) Molecular genetic basis of sudden cardiac death. Pediatr Clin North Am 51(5):1229-1255

28. Tsuchiya T, Ashikaga K, Honda T, Arita M (2002) Prevention of ventricular fibrillation by cilostazol, an oral phosphodiesterase inhibitor, in a patient with Brugada syndrome. J Cardiovasc Electrophysiol 13(7):698-701

29. Wilde AA, Antzelevitch C, Borggrefe M, Brugada J, Brugada R, Brugada P, Corrado D, Hauer RN, Kass RS, Nademanee K, Priori SG, Towbin JA, Study Group on the Molecular Basis of Arrhythmias of the European Society of Cardiology (2002) Proposed diagnostic criteria for the Brugada syndrome: consensus report. Circulation 106(19):2514-2519

30. Yamakawa Y, Ishikawa T, Uchino K, Mochida Y, Ebina T, Sumita S, Kobayashi T, Matsushita K, Matsumoto K, Ohkusu Y, Nishizawa T, Takigiku K, Iwamoto M, Kimura K, Umemura S (2004) Prevalence of right bundle-branch block and right precordial ST-segment elevation (Brugada-type electrocardiogram) in Japanese children. Circ J 68(4):275-279

31. Yoshinaga M, Anan R, Nomura Y, Tanaka Y, Sarantuya J, Oku S, Nishi S, Kawano Y, Tei C, Arima K (2004) Prevalence and time of appearance of Brugada electrocardiographic pattern in young male adolescents from a three-year follow-up study. Am J Cardiol 94(9):1186-1189 